\title{
Glucocorticoid receptor polymorphisms modulate cardiometabolic risk factors in patients in long-term remission of Cushing's syndrome
}

\author{
Sean H. P. P. Roerink ${ }^{1}$ • M. A. E. M. Wagenmakers ${ }^{1}$ J. W. A. Smit ${ }^{1}$ \\ E. F. C. van Rossum ${ }^{2}$ - R. T. Netea-Maier ${ }^{1}$ - T. S. Plantinga ${ }^{1}$ - A. R. M. M. Hermus ${ }^{1}$
}

Received: 15 September 2015/Accepted: 1 February 2016/Published online: 13 February 2016

(c) The Author(s) 2016. This article is published with open access at Springerlink.com

\begin{abstract}
Context Glucocorticoid receptor (GR) polymorphisms modulate glucocorticoid (GC) sensitivity and are associated with altered metabolic profiles.

Objective To evaluate the presence of GR polymorphisms ( $B c l \mathrm{I}$ (rs41423247), N363S (rs56149945), ER22/ 23EK (rs6189/rs6190), and 9 $\beta$ (rs6198) and investigate their associations with metabolic alterations in patients in long-term remission of Cushing's syndrome (CS).

Design and setting Cross-sectional case-control study.

Patients and methods Sixty patients in long-term remission of CS were genotyped. Associations between GR polymorphisms and multiple vascular, body composition and metabolic parameters were investigated.

Main outcome measures Allelic frequencies of the polymorphisms and their associations with several cardiometabolic risk factors.

Results This study shows that carriers of the $9 \beta$ polymorphism have a higher systolic blood pressure and lower resistin levels. The GC sensitizing BclI polymorphism is associated with an adverse cardiometabolic risk factor profile: higher fat percentages of extremities and legs, higher serum leptin and E-selectin levels, and higher intima media thickness in carriers versus non-carriers.
\end{abstract}

Sean H. P. P. Roerink

sean.roerink@radboudumc.nl

1 Division of Endocrinology, Department of Medicine, Radboud University Medical Centre, Geert Grooteplein 8, PO Box 9101, 6500 HB Nijmegen, The Netherlands

2 Division of Endocrinology, Department of Internal Medicine, Erasmus University Medical Center Rotterdam,

's-Gravendijkwal 230, 3015 CE Rotterdam, The Netherlands
Conclusions The $9 \beta$ and BclI polymorphisms of the GR adversely affect the cardiometabolic profile in patients who are in remission after the treatment of CS. This suggests that genetically altered GC sensitivity modulates the longterm adverse cardiometabolic effects resulting from (endogenous) hypercortisolism.

Keywords Cushing's syndrome - Long-term remission · Glucocorticoid receptor polymorphisms · Genetic predisposition - Metabolic profile

\section{Introduction}

Cushing's syndrome (CS) is a disorder resulting from chronic exposure to increased levels of glucocorticoids (GC), frequently caused by an ACTH-producing pituitary adenoma [Cushing's disease (CD)] or by primary adrenal overproduction of cortisol [adrenal CS (ACS)] [1]. CS is associated with body composition changes, cardiometabolic abnormalities such as type 2 diabetes mellitus (T2DM), hypertension and dyslipidemia, and ultimately cardiovascular disease [2]. We [3] and others [4, 5] have shown that many adverse metabolic and cardiovascular characteristics, and body compositional changes persist after treatment, even after long-term remission. Although these adverse metabolic and cardiovascular characteristics are common in these patients, their incidence and severity vary among patients. This variation seems not to be explained by differences in cortisol excess or disease duration alone. Therefore, a variable sensitivity to GC possibly plays a role in modulating the effect of cortisol excess [6].

Several investigations in healthy subjects have shown that GR polymorphisms are associated with altered GC sensitivity and alterations in metabolic profiles and body 
composition. The BclI and N363S polymorphisms of the GR gene have been associated with enhanced sensitivity to GC, increased abdominal obesity, an adverse lipid profile, and hyperinsulinemia [7-10]. In contrast, the ER22/23EK GR polymorphism has been associated with GC resistance and a favorable metabolic profile and body composition $[11,12]$. The $9 \beta$ polymorphism is associated with increased expression and stabilization of the dominant-negative splice variant GR- $\beta$. Enhanced GR- $\beta$ expression results in greater inhibition of GR- $\alpha$ transcriptional activity, and as GR- $\alpha$ is the functional GR isoform, in relative GC resistance [13-15]. This polymorphism is associated with increased serum levels of inflammatory parameters and cardiovascular disease despite a more favorable lipid profile in men and body composition in women $[16,17]$.

The functional role of GR polymorphisms has been extensively studied in the general healthy population. In contrast, only a small number of studies have been performed on the functional role of GR polymorphisms in active CS and CS in remission. These few studies have found an association of the $9 \beta$ polymorphism with the risk of developing diabetes mellitus [18] and of the BclI polymorphism with increased skeletal GC sensitivity and worse cognitive performance $[19,20]$.

We hypothesize that differences between patients in the severity of the adverse metabolic and vascular profile after cure of CS are related to differences in GC sensitivity due to GC receptor polymorphisms. Therefore, we investigated the associations of these genetic variants with the presence/ persistence of the adverse metabolic and vascular profile and body composition after long-term remission of CS.

\section{Subjects and methods}

Sixty adult ( $>18$-year old) patients in long-term remission ( $>4$ years) of CS were recruited from the outpatient clinic of the department of internal medicine. Remission was defined as suppression of plasma cortisol to $\leq 50 \mathrm{nmol} / \mathrm{L}$ after $1 \mathrm{mg}$ dexamethasone overnight [21] or, if a patient had received radiotherapy of the pituitary gland, a 24-h urinary-free cortisol excretion of $<240 \mathrm{nmol} / 24 \mathrm{~h}$ for men or $<150 \mathrm{nmol} / 24 \mathrm{~h}$ for women (upper levels of normal-free cortisol excretion). Clinical history was collected and a physical examination, biochemical and hormonal evaluation, dual-energy X-ray absorptiometry scanning (DXA), and non-invasive vascular function measurements were performed in all subjects. All subjects were genotyped for the presence of four GR polymorphisms; $B c l$ I, N363S, ER22/23EK, and $9 \beta$.

Patients with untreated hormonal deficiencies, or hormonal deficiencies that had not been treated adequately in the last 4 years according to international standards, were excluded. Furthermore, patients with active malignancy or systemic therapy for malignancy in the past, auto-inflammatory diseases, and psychiatric pathology were excluded. Hypothyroidism was defined as free thyroxine (fT4) plasma concentrations $<8 \mathrm{pmol} / \mathrm{l}$ (reference range $8-22 \mathrm{pmol} / \mathrm{l}$ ). Testosterone deficiency in men was defined as early morning testosterone levels $<11 \mathrm{nmol} / 1$ (reference range $11-45 \mathrm{nmol} / \mathrm{l})$. In women, estrogen deficiency was defined as secondary hypogonadotropic hypogonadism or a postmenopausal state without the use of chronic estrogen substitution therapy. Growth hormone $(\mathrm{GH})$ deficiency was defined as a maximal $\mathrm{GH}$ response of $<15.3 \mathrm{mU} / \mathrm{l}$ during an insulin tolerance test (ITT), or as a maximal GH response of $<12.3 \mathrm{mU} / \mathrm{l}$ during an arginine/GHRH test [22]. Glucocorticoid deficiency was defined as a maximal cortisol response $<550 \mathrm{nmol} / 1$ during an ITT [23]. All patients underwent a new $1 \mathrm{mg}$ dexamethasone suppression test (or a 24-h urinary-free cortisol measurement in case of pituitary RT) before entering the study to confirm remission. All subjects were of Caucasian (Dutch) origin.

\section{Clinical history}

Clinical history included the etiology of CS, treatment strategies (surgery, radiotherapy, medication), treatment for coexisting hormonal deficiencies, co-morbidities, and smoking habits.

\section{Physical examination}

Anthropometric measurements including weight and height, supine systolic, and diastolic blood pressure [average of 10 measurements, every 3 min with an oscillometric sphygmomanometer (Criticon model 1846; Criticon Inc., Tampa, FL)] measured at 09:00AM.

\section{Biochemical evaluation}

Biochemical evaluation included plasma level measurements of fasting glucose, glycated hemoglobin (HbA1c), total cholesterol, triglycerides, high-density lipoprotein (HDL), low-density lipoprotein (LDL), and insulin. Insulin sensitivity was assessed by homeostasis model assessment (HOMA) [24]. Furthermore, serum adipokines adiponectin, leptin, and resistin were measured. In addition, a number of markers of vascular health were measured in serum; soluble vascular cell adhesion molecule-1 (VCAM-1), soluble intercellular adhesion molecule-1 (ICAM-1), plasminogen activator inhibitor-1 (PAI-1), and soluble E-selectin. Increased levels of these parameters are indicators of endothelial dysfunction and worse vascular health. 


\section{Vascular evaluation}

Carotid intima media thickness (IMT) was determined using an AU5 ultrasound machine (Esaote Biomedical) with a $7.5 \mathrm{MHz}$ linear array transducer. Measurement of IMT was performed off-line by the sonographer at the time of the examination, using semi-automatic edge-detection software (M'Ath ${ }^{\circledR}$ Std version 2.0, Metris). IMT was defined as the mean IMT of the four measured segments of the common carotid artery: far wall left, near wall left, far wall right, and near wall right.

\section{Body composition}

Total body DXA was performed using a Hologic QDR 4500 densitometer (Hologic, Bedford, MA). Standard procedures supplied by the manufacturer for scanning and analyses (using Hologic software version 12.1) were followed. Calibration procedures were performed every day using the appropriate phantoms provided by the manufacturer. Total body fat mass was determined and fat percentage was calculated as the total body fat mass in percent of body weight. Furthermore, trunk-, leg-, and extremities fat percentages were determined in order to discriminate between different body regions. Different regions were determined by manually placing regions of interest as defined by the manufacturers software manual. The trunk fat depot is defined as the region between two horizontal lines placed on the lower border of the head and the upper border of the iliac crest, and two vertical lines placed against the outer margins of the chest. These lines exclude the arms from the trunk. The leg fat depot is defined by two vertical lines placed against the outer margins op both legs. The pelvis is excluded from the legs by a line through the femoral neck. Leg fat percentage is depicted as the average of both legs. Extremity fat percentage is depicted as the average of both arms and both legs.

\section{Laboratory measurements}

Serum concentrations of leptin, resistin, PAI-1, sICAM-1, and soluble E-selectin were measured by Multiplex Fluorescent Bead Immunoassays (xMAP technology, Millipore, Billerica, MA) and a Bio-plex microbead analyzer (Luminex, Austin, TX) according to the manufacturers protocol. Serum concentrations of adiponectin and SVCAM1 were determined by enzyme-linked immunosorbent assays (R\&D Systems, Minneapolis, MN). Fasting plasma glucose, $\mathrm{HbA1c}$, insulin and total serum cholesterol, triglycerides, LDL-cholesterol, and HDL-cholesterol were measured by standard procedures. According to the manufacturer's information, intra-assay precision coefficients of variation for all laboratory measurements were equal or below $5 \%$.

\section{Genetic analyses}

DNA was isolated from whole blood using the Gentra Puregene isolation kit (Qiagen, Valencia, CA, USA), according to the manufacturers protocol. Genotyping for the four selected genetic variants of the glucocorticoid receptor (GR) gene (official gene name $\mathrm{NR} 3 \mathrm{Cl}$ ) was performed as follows. The presence of the $9 \beta$ (rs6198) and N363S (rs56149945) genetic variants was assessed by applying the predesigned TaqMan SNP assays C_8951023_10 and C_26841917_40, respectively, on a 7300 ABI Real-Time polymerase chain reaction (PCR) system (all from Life Technologies, Applied Biosystems, Foster City, CA).

For the detection of the GR ER22/23EK (rs6189/rs6190), genetic variant conventional PCR and Sanger sequencing analysis were performed with forward primer $5^{\prime}$-CTG-CCTCTT-ACT-AAT-CGG-ATC-A- $3^{\prime}$ and reverse primer $5^{\prime}$ AGA-GTG-AAA-CTG-CTT-TGG-ACA-G- $3^{\prime}$. To determine the GR BclI genotype (rs41423247), DNA was amplified with forward primer $5^{\prime}$-AAG-CAA-TGC-AGT-GAACAG-TGT-AC- $3^{\prime}$ and reverse primer $5^{\prime}$-AAC-AAT-TTTGGC-CAT-CAG-TTA-TC- ${ }^{\prime}$. Also these PCR products were subjected to Sanger sequencing analysis.

\section{Statistical analyses}

Data are expressed as mean $\pm 95 \%$ confidence intervals unless stated otherwise. Data distributions were analyzed using the Kolmogorov-Smirnov test, and logarithmic transformation was performed before statistical testing when appropriate. Based on data distribution, comparison of continuous variables was performed using Student's $t$ test or Mann-Whitney rank sum test. The associations between genotypes and outcome measurements were evaluated using analysis of covariance (ANCOVA) with genotypes as a factor and age, gender, and BMI as covariates. Categorical variables were analyzed using the $\chi^{2}$ test followed by Fisher's exact test if appropriate. Hardy-Weinberg equilibrium for all polymorphisms was determined using a $\chi^{2}$ test. Significance was set at a $P$ value of $<0.05$. Statistical analysis was performed using SPSS Software version 20.0 (SPSS, Inc., Chicago, IL).

\section{Results}

Forty-eight $(80 \%)$ subjects were female. Mean (SD) age was 50.7 (12.4) years with a mean (SD) BMI of 26.9 (5.3) $\mathrm{kg} / \mathrm{m}^{2}$. Mean (SD) duration of remission was $13.8(8.5)$ years. Nineteen $(31.7 \%)$ subjects were treated for hypertension, $4(6.7 \%)$ were treated for diabetes mellitus, and $12(20.0 \%)$ were treated for hypercholesterolemia. Complete subject characteristics of all subjects are depicted in 
Table 1. Allelic frequencies of the four polymorphisms are depicted in Table 2. All polymorphisms were in HardyWeinberg equilibrium. Because of low allele frequencies, the N363S and ER22/23EK polymorphisms were not included in the association analysis. The minor allele of the $9 \beta$ polymorphism showed a statistically significant association with higher systolic blood pressure $(P=0.007)$ and lower resistin levels $(P=0.027)$ in carriers (Table 3; Fig. 1a). No associations were detected for the other measured parameters. The minor allele of the BclI genotype showed statistically significant associations with higher mean IMT $(P=0.048)$, higher extremities fat percentage $(P=0.007)$, higher leg fat percentage $(P=$ $0.029)$, higher leptin level $(P=0.038)$, and higher soluble E-selectin level $(P=0.037)$ in carriers (Table 3; Fig. 1b). No associations with the other measures were detected in our CS patients (data not shown).

Table 1 Subject characteristics

\begin{tabular}{ll}
\hline & $(n=60)$ \\
\hline Gender $(n)$ : male/female & $12 / 48$ \\
Age in years: mean (SD) & $50.7(12.4)$ \\
Duration of remission: mean (SD) & $13.8(8.5)$ \\
BMI in kg/m ${ }^{2}:$ mean (SD) & $26.9(5.3)$ \\
Waist circumference in cm: mean (SD) & $91.8(14.7)$ \\
Smoking $(n)$ : yes/no & $14 / 45$ \\
Treatment modalities: $n(\%)$ & \\
Unilateral adrenalectomy & $20(33.3)$ \\
Bilateral adrenalectomy & $12(20.0)$ \\
Pituitary surgery & $38(63.3)$ \\
Pituitary radiotherapy & $13(21.7)$ \\
Hormonal deficiencies: $n(\%)$ & \\
Glucocorticoid deficiency* & $22(36.7)$ \\
Growth hormone deficiency* & $15(25.0)$ \\
Thyroid hormone deficiency* & $26(43.3)$ \\
Testosterone deficiency* & $6(10.0)$ \\
Mineralocorticoid deficiency* & $11(18.3)$ \\
Estrogen deficiency & $26(54.2)$ \\
Cardiometabolic co-morbidities: $n(\%)$ & \\
Hypertension** & $19(31.7)$ \\
Diabetes mellitus* & $4(6.7)$ \\
Hypercholesterolemia** & $12(20.0)$ \\
Cushing type: $n$ (\%) & \\
Pituitary & $40(66.7)$ \\
Adrenal & $20(33.3)$ \\
\hline
\end{tabular}

BMI body mass index, $C S$ Cushing's syndrome

* Adequately substituted according to international standards

** Actively treated for this co-morbidity
Table 2 Genotype distributions and allele frequencies of the GR gene polymorphisms in patients cured of Cushing's syndrome (CS)

\begin{tabular}{ll}
\hline & CS $(n=60)$ \\
\hline clI & \\
CC & $32(53 \%)$ \\
CG & $20(33 \%)$ \\
GG & $8(13 \%)$ \\
Allele frequency & 0.300 \\
$9 \beta$ (A3669G) & \\
AA & $40(67 \%)$ \\
AG & $19(32 \%)$ \\
GG & $1(2 \%)$ \\
Allele frequency & 0.175 \\
N363S & \\
AA & $55(92 \%)$ \\
AG & $5(8 \%)$ \\
GG & $0(0 \%)$ \\
Allele frequency & 0.042 \\
ER22/23EK & \\
GG & $55(92 \%)$ \\
GA & $5(8 \%)$ \\
AA & $0(0 \%)$ \\
Allele frequency & 0.042 \\
\hline
\end{tabular}

\section{Discussion}

This study investigated the prevalence of GR polymorphisms in patients in long-term remission of CS and the associations of these polymorphisms with metabolic, vascular, and body compositional characteristics. In this study, we found that carriers of the $9 \beta$ polymorphism have a higher systolic blood pressure and lower resistin levels and that the GC sensitizing $B c l$ I polymorphism is associated with a number of metabolic and vascular adverse effects: higher fat percentages of extremities and legs, higher serum leptin, E-selectin levels, and higher intima media thickness in carriers versus non-carriers.

It has been shown that in patients in long-term remission of CS, cardiovascular and metabolic risk remain increased $[3,5]$. In the general population, it has been demonstrated that cardiovascular and metabolic risk and body composition are affected by lifelong overactivation or relative inactivation of GC signaling due to GR polymorphisms [25]. Our observation that altered glucocorticoid sensitivity due to GR polymorphisms modulates cardiometabolic risk factors in cured CS patients is in line with these wellknown findings in the general population.

Remitted CS patients carrying the minor allele of the $9 \beta$ polymorphism had a higher systolic blood pressure, which is concordant with previous findings of an increase in 
(a)

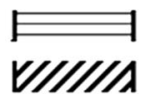

carrier

non-carrier

Systolic blood pressure

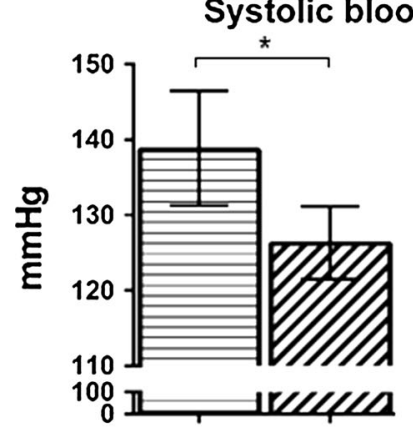

$*(P<0.05)$

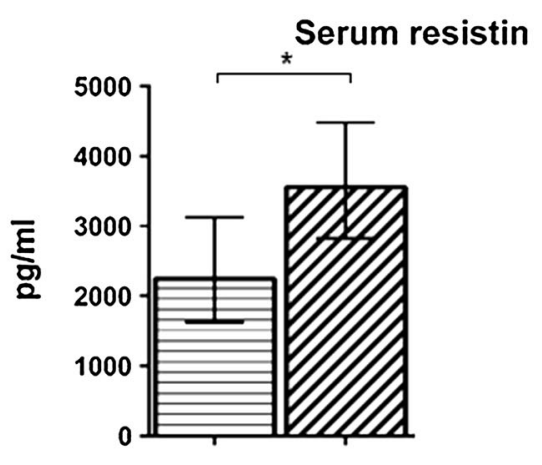

(b)
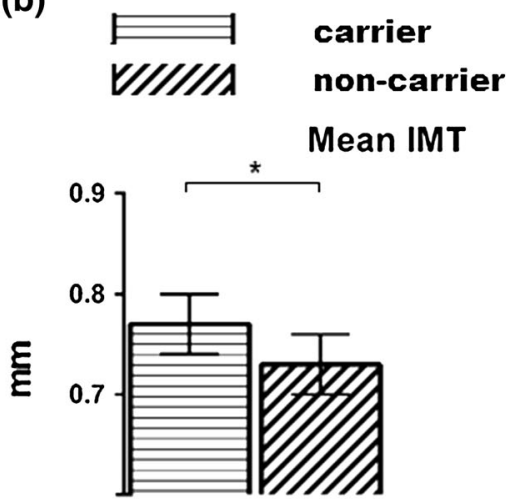

$0.1 \mathbb{1 2}$
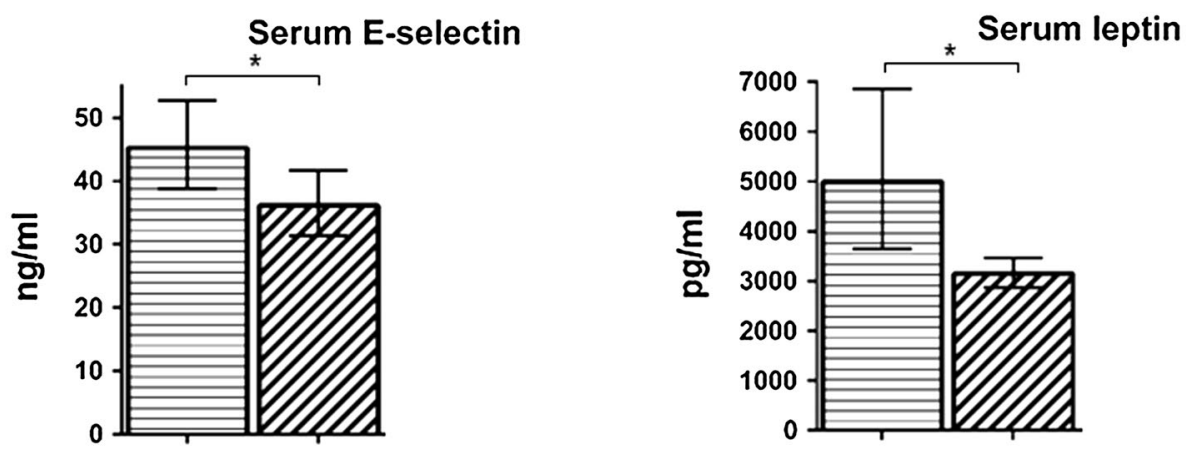
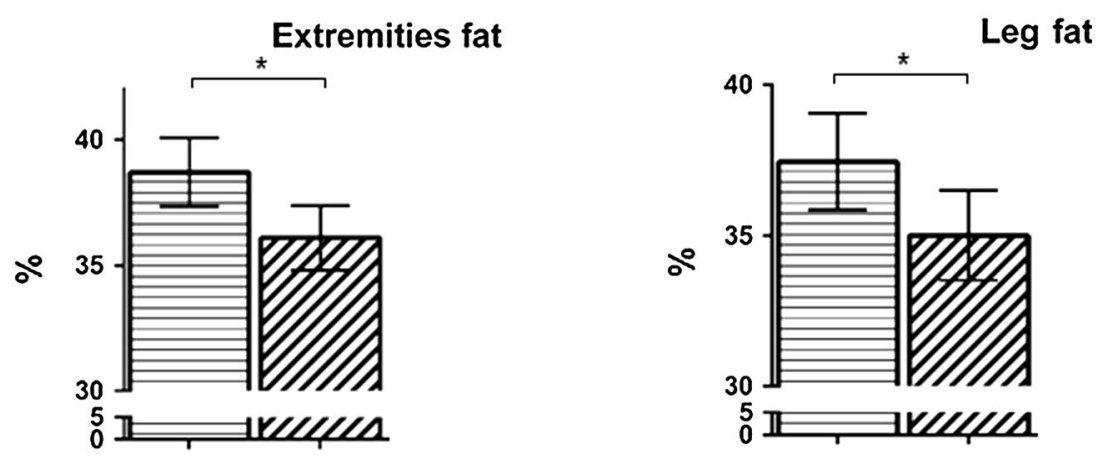

$*(P<0.05)$

Fig. 1 a Associations of the $9 \beta$ genotype and clinical outcome $* P<0.05$. b Associations of the $B c l$ I genotype and clinical outcome $* P<0.05$

carotid atherosclerosis, and a higher incidence of coronary heart disease in carriers of this polymorphism [17]. Systolic hypertension is normally especially seen in the elderly population due to increased arterial stiffening. Arterial stiffness is central to the pathogenesis of isolated systolic hypertension and directly impacts left ventricular afterload, pressure pulsatility in the arterial tree, and its penetration into the microvasculature of target organs such as the brain and kidney. This means that systolic hypertension in carriers of the polymorphism may lead to increased aging of the vascular tree, but further studies are needed to elucidate whether the polymorphism increases cardiovascular risk. Furthermore, an association was found between the $9 \beta$ polymorphism and resistin levels, with carriers of the minor allele of the $9 \beta$ polymorphism having lower resistin levels. It can be hypothesized that this is caused by the fact that subjects with a relative decrease in glucocorticoid sensitivity have less central adiposity. Central adiposity 
Table 3 Associations between glucocorticoid receptor polymorphisms and outcome measurements

\begin{tabular}{|c|c|c|c|}
\hline & Carrier minor allele & Non-carrier minor allele & $P$ value \\
\hline \multicolumn{4}{|l|}{$9 \beta$} \\
\hline Fasting glucose (mmol/l) & $5.1(4.7-5.4)$ & $5.0(4.7-5.2)$ & 0.578 \\
\hline $\mathrm{HbA} 1 \mathrm{c}(\mathrm{mmol} / \mathrm{mol})$ & $40.9(38.4-43.6)$ & $38.4(36.7-40.1)$ & 0.117 \\
\hline Total cholesterol (mmol/l) & $5.3(5.0-5.7)$ & $5.2(4.8-5.5)$ & 0.596 \\
\hline Triglycerides (mmol/l) & $1.4(1.2-1.8)$ & $1.5(1.3-1.7)$ & 0.850 \\
\hline HDL-cholesterol (mmol/l) & $1.3(1.2-1.5)$ & $1.4(1.3-1.4)$ & 0.919 \\
\hline LDL-cholesterol (mmol/l) & $3.2(2.9-3.6)$ & $3.1(2.9-3.3)$ & 0.498 \\
\hline Insulin $(\mathrm{mE} / \mathrm{l})$ & $6.0(4.6-7.8)$ & $7.2(6.0-8.7)$ & 0.266 \\
\hline HOMA-IR & $1.4(1.1-1.9)$ & $1.6(1.3-2.0)$ & 0.574 \\
\hline Mean IMT (mm) & $0.73(0.70-0.77)$ & $0.76(0.73-0.78)$ & 0.254 \\
\hline Systolic blood pressure $(\mathrm{mmHg})$ & $138.7(131.2-146.5)$ & $126.2(121.5-131.1)$ & $0.007 *$ \\
\hline Total body fat $(\%)$ & $32.6(30.8-34.3)$ & $34.3(33.1-35.2)$ & 0.114 \\
\hline Trunk fat $(\%)$ & $32.3(30.4-34.3)$ & $33.2(31.8-34.5)$ & 0.471 \\
\hline Extremities fat (\%) & $35.8(34.2-37.5)$ & $38.1(36.9-39.2)$ & 0.340 \\
\hline Leg fat $(\%)$ & $34.0(32.1-36.1)$ & $35.4(34.0-36.9)$ & 0.271 \\
\hline Leptin $(\mathrm{pg} / \mathrm{ml})$ & $3374.5(2289.3-4974.1)$ & 4213.3 (3206.7-5535.9) & 0.356 \\
\hline Resistin (pg/ml) & $2248.5(1618.1-3124.4)$ & $3558.2(2824.3-4482.8)$ & $0.020 *$ \\
\hline Adiponectin (pg/ml) & $706.3(458.1-1089.0)$ & $1087.9(802.7-1474.4)$ & 0.108 \\
\hline PAI-1 (pg/ml) & $1884.7(1377.2-2392.3)$ & 2384.8 (2027.4-2742.2) & 0.114 \\
\hline VCAM-1 (pg/ml) & $666.5(576.5-770.5)$ & $674.5609 .7-746.2)$ & 0.888 \\
\hline ICAM-1 (pg/ml) & $253.4(177.0-362.9)$ & $304.3(236.3-391.9)$ & 0.410 \\
\hline E-selectin (pg/ml) & $46.2(37.9-54.5)$ & $42.2(36.3-48.1)$ & 0.436 \\
\hline \multicolumn{4}{|l|}{$B c l \mathrm{I}$} \\
\hline Fasting glucose $(\mathrm{mmol} / \mathrm{l})$ & $5.2(4.9-5.5)$ & $4.8(4.6-5.1)$ & 0.097 \\
\hline $\mathrm{HbA} 1 \mathrm{c}(\mathrm{mmol} / \mathrm{mol})$ & $40.6(38.5-42.8)$ & $38.1(36.2-40.0)$ & 0.089 \\
\hline Total cholesterol (mmol/l) & $5.2(4.9-5.6)$ & $5.2(4.9-5.5)$ & 0.837 \\
\hline Triglycerides (mmol/l) & $1.6(1.4-1.9)$ & $1.3(1.1-1.6)$ & 0.198 \\
\hline HDL-cholesterol (mmol/l) & $1.4(1.2-1.5)$ & $1.4(1.3-1.4)$ & 0.969 \\
\hline LDL-cholesterol (mmol/l) & $3.1(2.9-3.4)$ & $3.2(2.9-3.4)$ & 0.879 \\
\hline Insulin (mE/l) & $7.4(5.9-9.2)$ & $6.3(5.1-7.8)$ & 0.313 \\
\hline HOMA-IR & $1.8(1.4-2.3)$ & $1.4(1.1-1.7)$ & 0.125 \\
\hline Mean IMT (mm) & $0.77(0.74-0.80)$ & $0.73(0.70-0.76)$ & $0.048 *$ \\
\hline Systolic blood pressure $(\mathrm{mmHg})$ & $126.0(120.2-132.0)$ & $134.0(128.3-14 .-1)$ & 0.060 \\
\hline Total body fat $(\%)$ & $34.5(33.0-36.0)$ & $33.1(31.7-34.4)$ & 0.167 \\
\hline Trunk fat $(\%)$ & $33.3(31.7-34.9)$ & $32.5(31.0-34.1)$ & 0.488 \\
\hline Extremities fat $(\%)$ & $38.7(37.3-40.1)$ & $36.1(34.8-37.4)$ & $0.007 *$ \\
\hline Leg fat $(\%)$ & $37.5(35.8-39.1)$ & $35.0(33.5-36.5)$ & $0.029 *$ \\
\hline Leptin (pg/ml) & $4999.0(3644.6-6856.8)$ & $3155.8(2869.8-3470.3)$ & 0.038* \\
\hline Resistin (pg/ml) & $3630.0(2738.1-4812.6)$ & $2625.4(2016.3-3418.7)$ & 0.099 \\
\hline Adiponectin (pg/ml) & $1166.8(811.6-1677.4)$ & $781.3(556.1-1097.7)$ & 0.112 \\
\hline PAI-1 (pg/ml) & $2301.6(1866.2-2737.1)$ & $2145.0(1737.8-2552.2)$ & 0.602 \\
\hline VCAM-1 (pg/ml) & $726.3(645.5-817.3)$ & $627.7(562.3-700.6)$ & 0.075 \\
\hline ICAM-1 (pg/ml) & $282.3(208.5-382.2)$ & $289.5(218.1-384.1)$ & 0.904 \\
\hline E-selectin (ng/ml) & $45.2(38.8-52.7)$ & $36.1(31.3-41.7)$ & $0.037 *$ \\
\hline
\end{tabular}

Data are expressed as mean and $95 \%$ confidence intervals

All data are adjusted for age, gender, and BMI

$I M T$ intima media thickness, $H b A l c$ glycated hemoglobin, $H D L$ high-density lipoprotein, $L D L$ low-density lipoprotein, HOMA-IR homeostatic model assessment-insulin resistance, PAI plasminogen activator inhibitor, VCAM vascular cell adhesion molecule, ICAM intracellular adhesion molecule

* $P$ ANCOVA was used to assess the statistical differences between genotype groups, $P<0.05$ (bold) 
causes local ischemia of the adipose tissue which leads to infiltration of M1-macrophages, which are the main producers of resistin. This hypothesis is, however, speculative and not supported by the data, since no association between truncal fat and the $9 \beta$ polymorphism was observed.

The minor allele of the BclI polymorphism was associated with an increased fat percentage of the extremities and legs in the remitted CS patients. These body areas only contain subcutaneous adipose tissue. As leptin is preferentially produced in subcutaneous adipose tissue (SAT), carriers also had higher leptin levels [26]. High leptin levels have been widely recognized as an independent cardiovascular risk factor associated with insulin resistance. It also has a pathogenic role in atherothrombosis and endothelial dysfunction. Furthermore, a higher level of E-selectin was found in carriers of the minor allele of the $B c l$ I polymorphism. This may reflect increased endothelial activation and progressing atherosclerosis in the BclI carriers. This is supported by our observation that the IMT is higher in BclI carriers.

In women with CS in remission, the $B c l$ I polymorphism was previously shown to be associated with altered GC sensitivity as the polymorphism was associated with reduced total and femoral neck bone mineral density [27]. Furthermore, in patients in remission of CS, the BclI polymorphism was also independently associated with increased fatigue and worse performance on cognitive testing [20]. The findings in literature combined with the findings in the current study suggest that hypercortisolism in patients carrying the $B c l$ I polymorphism may have more pronounced effects on patient wellbeing in the long term.

This study has some limitations. Because of the low incidence of CS, only a relatively small cohort could be studied. This could influence genetic associations and that is why only polymorphisms with a relatively high frequency were included in the association analysis. In genetic association studies, inhomogeneity of study population with regard to ethnicity, gender, age, and environmental factors is a frequent limiting factor. This study included Caucasians only and associations were corrected for gender and age. Duration of hypercortisolism likely represents another factor in long-term cardiometabolic effects in CS patients, which, however, is rather difficult to estimate since diagnosis of CS is generally delayed. Furthermore, it was decided not to correct for multiple testing because of the hypothesis-driven nature of this study. Indeed, from a biological perspective our findings are in line with the expected corticosteroid effects, suggesting that SNPs of the GR gene leading to either hypo- or hypersensitivity to GC, indeed modulate long-term cardiometabolic outcome after treatment of CS. However, because of the limited sample size and, hence, limited statistical power, we cannot exclude that some of the significant differences between carriers and non-carriers may be a finding by chance, i.e., a type-1 error.

In conclusion, this study is one of the first to suggest that glucocorticoid receptor polymorphisms modulate cardiometabolic risk factors in patients in long-term remission of CS [27]. In the context of personalized healthcare, this may implicate that, after treatment of CS, carriers of these polymorphisms are candidates for a more stringent followup regarding cardiovascular and metabolic health as our findings suggest that GR polymorphisms may play a role in susceptibility to cardiovascular disease in CS patients. Furthermore, patients who treated with glucocorticoids for other diseases and even healthy subjects, carrying these polymorphisms, are candidates for a more stringent followup regarding cardiovascular and metabolic health, and the development of metabolic syndrome as was recently debated in literature [28]. However, the results of this study need to be interpreted with caution, and further research into these findings in larger CS populations is needed to replicate these findings. In addition, future studies should delineate to what extent the observed associations also apply to prolonged episodes of exposure to physiologically elevated cortisol levels (e.g., severe stress) or exogenous glucocorticoids.

Funding This work was financially supported by the Dutch Adrenal Patient Society (NVACP) and the Dutch Adrenal Fund.

\section{Compliance with ethical standards}

Conflict of interest The authors declare that there is no conflict of interest that could be perceived as prejudicing the impartiality of the research reported.

Open Access This article is distributed under the terms of the Creative Commons Attribution 4.0 International License (http://crea tivecommons.org/licenses/by/4.0/), which permits unrestricted use, distribution, and reproduction in any medium, provided you give appropriate credit to the original author(s) and the source, provide a link to the Creative Commons license, and indicate if changes were made.

\section{References}

1. J. Lindholm, S. Juul, J.O. Jorgensen, J. Astrup, P. Bjerre, U. Feldt-Rasmussen, C. Hagen, J. Jorgensen, M. Kosteljanetz, L. Kristensen, P. Laurberg, K. Schmidt, J. Weeke, Incidence and late prognosis of cushing's syndrome: a population-based study. J. Clin. Endocrinol. Metab. 86(1), 117-123 (2001)

2. G. Arnaldi, A. Angeli, A.B. Atkinson, X. Bertagna, F. Cavagnini, G.P. Chrousos, G.A. Fava, J.W. Findling, R.C. Gaillard, A.B. Grossman, B. Kola, A. Lacroix, T. Mancini, F. Mantero, J. Newell-Price, L.K. Nieman, N. Sonino, M.L. Vance, A. Giustina, M. Boscaro, Diagnosis and complications of Cushing's syndrome: a consensus statement. J. Clin. Endocrinol. Metab. 88(12), 5593-5602 (2003) 
3. M. Wagenmakers, S. Roerink, L. Gil, T. Plantinga, J. Smit, R. Netea-Maier, A. Hermus, Persistent centripetal fat distribution and metabolic abnormalities in patients in long-term remission of Cushing's syndrome. Clin. Endocrinol. 82(2), 180-187 (2015). doi:10.1111/cen.12639

4. M.J. Barahona, N. Sucunza, E. Resmini, J.M. Fernandez-Real, W. Ricart, J.M. Moreno-Navarrete, T. Puig, J. Farrerons, S.M. Webb, Persistent body fat mass and inflammatory marker increases after long-term cure of Cushing's syndrome. J. Clin. Endocrinol. Metab. 94(9), 3365-3371 (2009). doi:10.1210/jc.2009-0766

5. A. Colao, R. Pivonello, S. Spiezia, A. Faggiano, D. Ferone, M. Filippella, P. Marzullo, G. Cerbone, M. Siciliani, G. Lombardi, Persistence of increased cardiovascular risk in patients with Cushing's disease after five years of successful cure. J. Clin. Endocrinol. Metab. 84(8), 2664-2672 (1999). doi:10.1210/jcem. 84.8.5896

6. N.A. Huizenga, J.W. Koper, P. de Lange, H.A. Pols, R.P. Stolk, D.E. Grobbee, F.H. de Jong, S.W. Lamberts, Interperson variability but intraperson stability of baseline plasma cortisol concentrations, and its relation to feedback sensitivity of the hypothalamo-pituitary-adrenal axis to a low dose of dexamethasone in elderly individuals. J. Clin. Endocrinol. Metab. 83(1), 47-54 (1998)

7. E.F. van Rossum, S.W. Lamberts, Polymorphisms in the glucocorticoid receptor gene and their associations with metabolic parameters and body composition. Recent Prog. Horm. Res. 59, 333-357 (2004)

8. N.A. Huizenga, J.W. Koper, P. De Lange, H.A. Pols, R.P. Stolk, H. Burger, D.E. Grobbee, A.O. Brinkmann, F.H. De Jong, S.W. Lamberts, A polymorphism in the glucocorticoid receptor gene may be associated with and increased sensitivity to glucocorticoids in vivo. J. Clin. Endocrinol. Metab. 83(1), 144-151 (1998)

9. A.M. Di Blasio, E.F. van Rossum, S. Maestrini, M.E. Berselli, M. Tagliaferri, F. Podesta, J.W. Koper, A. Liuzzi, S.W. Lamberts, The relation between two polymorphisms in the glucocorticoid receptor gene and body mass index, blood pressure and cholesterol in obese patients. Clin. Endocrinol. 59(1), 68-74 (2003)

10. E.F. van Rossum, J.W. Koper, A.W. van den Beld, A.G. Uitterlinden, P. Arp, W. Ester, J.A. Janssen, A.O. Brinkmann, F.H. de Jong, D.E. Grobbee, H.A. Pols, S.W. Lamberts, Identification of the BclI polymorphism in the glucocorticoid receptor gene: association with sensitivity to glucocorticoids in vivo and body mass index. Clin. Endocrinol. 59(5), 585-592 (2003)

11. E.F. van Rossum, J.W. Koper, N.A. Huizenga, A.G. Uitterlinden, J.A. Janssen, A.O. Brinkmann, D.E. Grobbee, F.H. de Jong, C.M. van Duyn, H.A. Pols, S.W. Lamberts, A polymorphism in the glucocorticoid receptor gene, which decreases sensitivity to glucocorticoids in vivo, is associated with low insulin and cholesterol levels. Diabetes 51(10), 3128-3134 (2002)

12. E.F. van Rossum, P.G. Voorhoeve, S.J. te Velde, J.W. Koper, H.A. Delemarre-van de Waal, H.C. Kemper, S.W. Lamberts, The ER22/23EK polymorphism in the glucocorticoid receptor gene is associated with a beneficial body composition and muscle strength in young adults. J. Clin. Endocrinol. Metab. 89(8), 4004-4009 (2004). doi:10.1210/jc.2003-031422

13. R.H. Oakley, C.M. Jewell, M.R. Yudt, D.M. Bofetiado, J.A. Cidlowski, The dominant negative activity of the human glucocorticoid receptor beta isoform. Specificity and mechanisms of action. J. Biol. Chem. 274(39), 27857-27866 (1999)

14. R.H. Oakley, M. Sar, J.A. Cidlowski, The human glucocorticoid receptor beta isoform. Expression, biochemical properties, and putative function. J. Biol. Chem. 271(16), 9550-9559 (1996)

15. E.L. van den Akker, H. Russcher, E.F. van Rossum, A.O. Brinkmann, F.H. de Jong, A. Hokken, H.A. Pols, J.W. Koper, S.W. Lamberts, Glucocorticoid receptor polymorphism affects transrepression but not transactivation. J. Clin. Endocrinol. Metab. 91(7), 2800-2803 (2006). doi:10.1210/jc.2005-2119

16. A.A. Syed, J.A. Irving, C.P. Redfern, A.G. Hall, N.C. Unwin, M. White, R.S. Bhopal, J.U. Weaver, Association of glucocorticoid receptor polymorphism A3669G in exon 9beta with reduced central adiposity in women. Obesity 14(5), 759-764 (2006). doi:10.1038/oby.2006.86

17. E.L. van den Akker, J.W. Koper, E.F. van Rossum, M.J. Dekker, H. Russcher, F.H. de Jong, A.G. Uitterlinden, A. Hofman, H.A. Pols, J.C. Witteman, S.W. Lamberts, Glucocorticoid receptor gene and risk of cardiovascular disease. Arch. Intern. Med. 168(1), 33-39 (2008). doi:10.1001/archinternmed.2007.41

18. L. Trementino, G. Appolloni, C. Concettoni, M. Cardinaletti, M. Boscaro, G. Arnaldi, Association of glucocorticoid receptor polymorphism A3669G with decreased risk of developing diabetes in patients with Cushing's syndrome. Eur.J. Endocrinal. 166(1), 35-42 (2012). doi:10.1530/EJE-11-0722

19. A. Szappanos, A. Patocs, J. Toke, B. Boyle, M. Sereg, J. Majnik, G. Borgulya, I. Varga, I. Liko, K. Racz, M. Toth, BclI polymorphism of the glucocorticoid receptor gene is associated with decreased bone mineral density in patients with endogenous hypercortisolism. Clin. Endocrinol. 71(5), 636-643 (2009). doi:10.1111/j.1365-2265.2009.03528.x

20. O. Ragnarsson, C. Glad, P. Berglund, R. Bergthorsdottir, D.N. Eder, G. Johannsson, Common genetic variants in the glucocorticoid receptor and the 11beta-Hydroxysteroid dehydrogenase type 1 genes influence long-term cognitive impairments in patients with Cushing's syndrome in remission. J. Clin. Endocrinol. Metab. (2014). doi:10.1210/jc.2014-1906

21. L.K. Nieman, B.M. Biller, J.W. Findling, J. Newell-Price, M.O. Savage, P.M. Stewart, V.M. Montori, The diagnosis of Cushing's syndrome: an endocrine society clinical practice guideline. J. Clin. Endocrinol. Metab. 93(5), 1526-1540 (2008). doi:10. 1210/jc.2008-0125

22. M.E. Molitch, D.R. Clemmons, S. Malozowski, G.R. Merriam, S.M. Shalet, M.L. Vance, S. Endocrine Society's Clinical Guidelines, P.A. Stephens, Evaluation and treatment of adult growth hormone deficiency: an endocrine society clinical practice guideline. J. Clin. Endocrinol. Metab. 91(5), 1621-1634 (2006). doi:10.1210/jc.2005-2227

23. W. Arlt, B. Allolio, Adrenal insufficiency. Lancet 361(9372), 1881-1893 (2003). doi:10.1016/S0140-6736(03)13492-7

24. D.R. Matthews, J.P. Hosker, A.S. Rudenski, B.A. Naylor, D.F. Treacher, R.C. Turner, Homeostasis model assessment: insulin resistance and beta-cell function from fasting plasma glucose and insulin concentrations in man. Diabetologia 28(7), 412-419 (1985)

25. R. Pivonello, A. Faggiano, G. Lombardi, A. Colao, The metabolic syndrome and cardiovascular risk in Cushing's syndrome. Endocrinol. Metab. Clin. North Am. 34(2), 327-339 (2005). doi:10.1016/j.ecl.2005.01.010. viii

26. B.L. Wajchenberg, D. Giannella-Neto, M.E. da Silva, R.F. Santos, Depot-specific hormonal characteristics of subcutaneous and visceral adipose tissue and their relation to the metabolic syndrome. Horm Metab Res 34(11-12), 616-621 (2002). doi:10. 1055/s-2002-38256

27. O. Ragnarsson, C.A. Glad, R. Bergthorsdottir, E.G. Almqvist, E. Ekerstad, H. Widell, B. Wangberg, G. Johannsson, Body composition and bone mineral density in women with Cushing's syndrome in remission and the association with common genetic variants influencing glucocorticoid sensitivity. Eur. J. Endocrinol. 172(1), 1-10 (2015). doi:10.1530/EJE-14-0747

28. Y.X. Yan, J. Dong, J. Zhang, F. Liu, W. Wang, L. Zhang, Y. He, Polymorphisms in NR3C1 gene associated with risk of metabolic syndrome in a Chinese population. Endocrine 47(3), 740-748 (2014). doi:10.1007/s12020-014-0324-9 